\title{
Perfil nutricional, clínico e socioeconômico de pacientes com fibrose cística atendidos em um centro de referência no nordeste do Brasil*
}

\author{
Nutritional, clinical and socioeconomic profile of patients with \\ cystic fibrosis treated at a referral center in northeastern Brazil \\ lsabel Carolina da Silva Pinto, Cristiane Pereira da Silva, \\ Murilo Carlos Amorim de Britto
}

\begin{abstract}
Resumo
Objetivo: Descrever o perfil de pacientes portadores de fibrose cística (FC). Métodos: Estudo transversal, prospectivo, avaliando fibrocísticos de $\leq 18$ anos, durante o período de março a julho de 2006, em um centro de referência no nordeste do Brasil. A avaliação nutricional foi realizada pelo escore $\mathrm{Z}$ de altura/idade (A/1), peso/ idade $(\mathrm{P} / \mathrm{l})$ e peso/altura $(\mathrm{P} / \mathrm{A})$ e \%peso/altura $(\% \mathrm{P} / \mathrm{A})$, além de medidas de composição corporal. Foram obtidos dados socioeconômicos e clínicos. Resultados: Foram avaliados 21 pacientes, sendo $12(57,1 \%)$ do sexo feminino. A média de idade de diagnóstico foi de 3,8 \pm 3,9 anos, e as principais características ao diagnóstico foram infecção respiratória (85,7\%), esteatorreia (66,7\%) e déficit nutricional (47,6\%). A média de escore $Z$ para P/1, A/1 e $\mathrm{P} / \mathrm{A}$, respectivamente, foi de $-0,73 \pm 0,28,-0,34 \pm 0,21$ e $-0,73 \pm 0,35$. A média de $\% \mathrm{P} / \mathrm{A}$ foi de $94,52 \pm 1,58$. 0 percentual de desnutridos divergiu quando avaliado pelo escore $\mathrm{Z}$ e \%P/A (déficit nutricional em 66,7\% e 33,3\%, respectivamente; $p>0,05)$. Os pacientes eutróficos apresentaram melhores condições socioeconômicas $(p>0,05)$ e clínicas, com melhor escore de Shwachman $(p<0,05)$ quando comparados aos distróficos. Conclusões: Eutrofia foi encontrada através dos indicadores nutricionais ( $\mathrm{P} / 1, \mathrm{~A} / 1$ e $\mathrm{P} / \mathrm{A})$, ao passo que déficit nutricional foi encontrado quando avaliada a composição corporal. As condições socioeconômicas apresentaram-se favoráveis, principalmente em relação ao grau de instrução materna e renda per capita. A idade de diagnóstico foi maior do que o relatado na literatura, embora o escore de Shwachman e o número de infecções respiratórias tenham demonstrado que os pacientes tinham boas condições clínicas.
\end{abstract}

Descritores: Fibrose cística; Avaliação nutricional; Sintomas clínicos; Condições socioeconômicas.

\begin{abstract}
Objective: To describe the profile of patients with cystic fibrosis (CF). Methods: A prospective, cross-sectional study involving CF patients $\leq 18$ years of age, evaluated between March and July of 2006 at a referral center in northeastern Brazil. Nutritional assessment was performed using Z scores for height/age (H/A), weight/age (W/A) and weight/height $(\mathrm{W} / \mathrm{H})$, as well as \%weight/height $(\% \mathrm{~W} / \mathrm{H})$ and body composition measurements. Socioeconomic and clinical data were obtained. Results: Twenty-one patients were evaluated, $12(57.1 \%)$ of whom were female. Mean age at diagnosis was $3.8 \pm 3.9$ years. The principal features at diagnosis were respiratory infection (85.7\%), steatorrhea (66.7\%) and nutritional deficit (47.6\%). The mean Z scores for W/A, H/A and W/H were $0.73 \pm 0.28$, $0.34 \pm 0.21$ and $0.73 \pm 0.35$, respectively. Mean $\% \mathrm{~W} / \mathrm{H}$ was $94.52 \pm 1.58$. The percentage of malnourished children assessed by $\mathrm{Z}$ score differed from that assessed by \%W/H (nutritional deficit in 66.7\% and 33.3\%, respectively; $\mathrm{p}>$ 0.05). Socioeconomic status, clinical status and Shwachman score were better among well-nourished patients than among those classified as malnourished ( $p<0.05$ for Shwachman score). Conclusions: Normal nutritional status was identified based on nutritional indicators (W/A, H/A and W/H), whereas nutritional deficit was identified by assessing body composition. Socioeconomic factors proved favorable, especially maternal education and per capita income. Age at diagnosis was higher than that reported in the literature, although the Shwachman score and the incidence of respiratory infections demonstrated that the patients presented good clinical status.
\end{abstract}

Keywords: Cystic fibrosis; Nutritional assessment; Symptoms, clinical; Socioeconomic factors.

\footnotetext{
* Trabalho realizado no Instituto Materno Infantil Professor Fernando Figueira - IMIP - Recife (PE) Brasil.

Endereço para correspondência: Isabel Carolina da Silva Pinto. Departamento de Nutrição, Rua dos Coelhos, 300, Boa Vista, CEP 50070-550, Recife, PE, Brasil.

Tel/Fax 5581 2122-4120. E-mail: isabelcspinto@yahoo.com.br

Apoio financeiro: Nenhum.

Recebido para publicação em 11/2/2008. Aprovado, após revisão, em 11/8/2008.
} 


\section{Introdução}

A fibrose cística (FC) é uma desordem autossômica recessiva que afeta vários sistemas do corpo humano, caracterizando-se por uma disfunção generalizada das glândulas exócrinas e resultando em DPOC, com acúmulo de secreção espessa e purulenta, infecções respiratórias recorrentes, perda progressiva da função pulmonar, depuração mucociliar diminuída, insuficiência pancreática e aumento de eletrólitos no suor, bem como acometimento, com menor frequência, do intestino, fígado, vias biliares e genitais. ${ }^{(1,2)}$

No Brasil, estima-se que a prevalência da doença seja de 1:10.000 nascidos vivos, embora haja variação na frequência das mutações em diferentes regiões geográficas. Na região sul, essa prevalência se aproxima da população caucasiana centro-europeia (de 1:2.000 a 1:5.000 nascidos vivos). ${ }^{(1,3,4)}$

Nas últimas décadas, os conhecimentos acerca das mutações genéticas e o desenvolvimento de técnicas diagnósticas cada vez mais precoces têm melhorado bastante o prognóstico e a qualidade de vida dos pacientes com FC. No entanto, a desnutrição e o déficit de crescimento nesses pacientes continuam atingindo prevalências significantes. Nos EUA, cerca de $20 \%$ das crianças e adolescentes com FC apresentaram peso e estatura abaixo do percentil $5 .{ }^{(5)}$

Já é bem estabelecida na literatura a íntima relação existente entre alterações da composição corporal, desnutrição e função pulmonar. ${ }^{(1)} \mathrm{A}$ desnutrição afeta negativamente a função dos músculos respiratórios, a elasticidade pulmonar e a resposta imune, por aumentar o risco de colonizações pulmonares, principalmente quando associadas à diminuição da massa magra. ${ }^{(6)}$

Os problemas nutricionais apresentados pelos fibrocísticos são multifatoriais e podem ser atribuídos ao desequilíbrio energético decorrente da doença respiratória crônica, má absorção dos nutrientes causada pela insuficiência pancreática, alterações na circulação entero-hepática de sais biliares e antibioticoterapia, além de poder ser agravada pela presença de anorexia decorrente de refluxo gastroesofágico ou tosse, metabolismo aumentado, principalmente na presença de infecções, e estresse psicossocial..$^{(7,8)}$

0 objetivo deste estudo foi descrever o perfil nutricional, clínico e socioeconômico de pacientes portadores de FC acompanhados em um centro de referência no nordeste do Brasil, visto a escassez de estudos avaliando tais condições em pacientes dessa região.

\section{Métodos}

Realizou-se um estudo transversal, prospectivo, desenvolvido no Ambulatório de Pediatria no Serviço de Doenças Respiratórias do Instituto Materno Infantil Professor Fernando Figueira (IMIP), Recife, Brasil, no período de março a julho de 2006. Foram convidados a participar do estudo todas as crianças e adolescentes com diagnóstico confirmado de FC e idade $\leq 18$ anos que compareceram no ambulatório durante o período de estudo. Não houve exclusão de nenhum deles.

0 diagnóstico da $\mathrm{FC}$ foi realizado na presença de pelo menos uma das manifestações clínicas características da doença e por no mínimo duas dosagens de cloretos no suor por iontoforese com pilocarpina com valores acima de $60 \mathrm{mEq} / \mathrm{L}$ ou pela presença de duas mutações conhecidas para FC através de estudo genético. ${ }^{(9)}$

Para a avaliação socioeconômica, utilizou-se um questionário estruturado contendo dados de identificação materna (idade, escolaridade e paridade), número de residentes no domicílio e renda mensal per capita. Em relação à procedência, baseado na cidade a qual a família

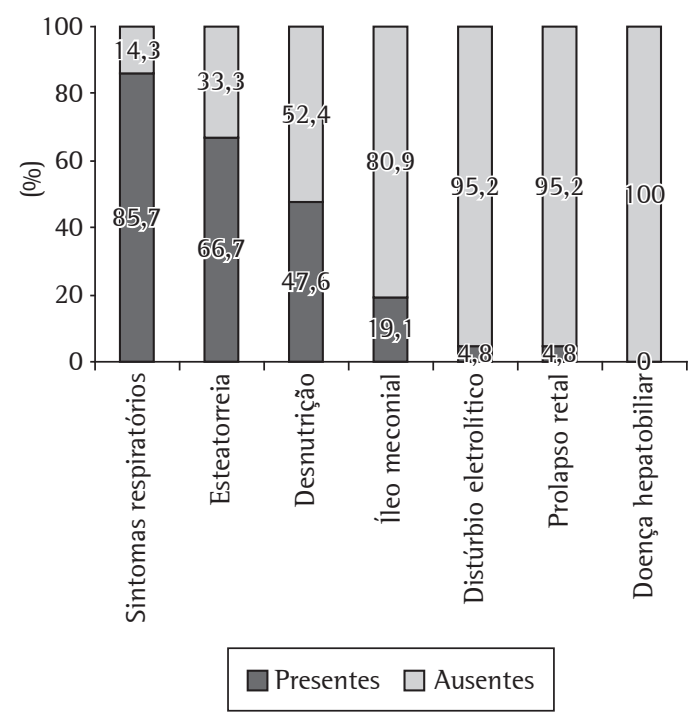

Figura 1 - Manifestações clínicas presentes ao diagnóstico em fibrocísticos atendidos em um centro de referência no Recife (PE). 
habita, os pacientes foram agrupados em três regiões: região metropolitana do Recife, interior do estado de Pernambuco e outros estados.

Foram coletados dados sobre a idade gestacional dos pacientes definindo como pré-termo ( $<37$ semanas) e a termo ( $\geq 37$ semanas); peso ao nascer, sendo baixo peso $(<2.500 \mathrm{~g})$ e adequado $(\geq 2.500 \mathrm{~g})$; e tempo de aleitamento materno exclusivo.

Para a avaliação clínica, observou-se a idade de diagnóstico e as manifestações clínicas presentes ao diagnóstico. A avaliação da gravidade clínica da doença baseou-se no escore de Shwachman realizado pelo pneumopediatra, que avaliou, em pontos com pesos iguais, os seguintes itens: atividade geral, quadro pulmonar, achados radiológicos e condição nutricional. Cada critério varia de 5 a 25 pontos, e quanto menor a pontuação maior é a gravidade. ${ }^{(10)}$ Foi considerada como colonização pulmonar quando o indivíduo apresentou cultura positiva para Pseudomonas aeruginosa, Staphylococcus aureus (oxacilina sensível e resistente) e Burkholderia cepacia.

Para a avaliação do estado nutricional, os indivíduos foram divididos em três faixas etárias: menores de 5 anos, entre 5 e 10 anos e maiores de 10 anos. Utilizaram-se medidas de peso, estatura, circunferência do braço (CB) e prega cutânea tricipital (PCT). Nas crianças menores de 2 anos, foi utilizada uma balança pediátrica com capacidade para $15 \mathrm{~kg}$ e precisão de $100 \mathrm{~g}$ (Filizola, São Paulo, Brasil) e um infantômetro para a medição do comprimento. Nas crianças maiores de 2 anos, foi utilizada uma balança digital com capacidade para $150 \mathrm{~kg}$ e precisão de $50 \mathrm{~g}$ (Filizola) e um estadiômetro para a aferição da estatura.
Essas medidas foram expressas em escore $\mathrm{Z}$ para peso/idade $(\mathrm{P} / \mathrm{l})$, altura/idade $(\mathrm{A} / \mathrm{l})$ e peso/ altura $(\mathrm{P} / \mathrm{A})$ baseados em idade e sexo, comparando-as com os padrões da Organização Mundial de Saúde (OMS), ${ }^{(11)}$ para os indivíduos menores de 5 anos, e do National Center for Health Statistics, ${ }^{(12)}$ para os maiores de 5 anos. Consideraram-se como portadores de déficit nutricional aqueles que apresentassem escore $\mathrm{Z}$ $<-1$ dp para qualquer indicador avaliado, e, como eutróficos, quando todos os indicadores fossem $\geq-1 \mathrm{dp}$. 0 percentual de peso ideal em relação à altura $(\% \mathrm{P} / \mathrm{A})$ também foi calculado conforme recomendado pelo US Cystic Fibrosis Foundation Consensus Report, o qual classifica em risco nutricional $<90 \%$ e eutrofia $\geq 90 \% .^{(13,14)}$

Para a aferição da CB, foi utilizada uma fita métrica inextensível. Para a aferição da PCT, utilizou-se um compasso de dobras cutâneas (Langer Skinfold Caliper; Cambridge Scientific Industries, Cambridge, MD, EUA), utilizado para crianças maiores de 6 anos. As medidas foram realizadas no ponto médio entre o olécrano e o acrômio no braço não-dominante do paciente.

Utilizando-se a CB e a PCT, avaliou-se a massa muscular e adiposa, calculando-se a área muscular do braço (AMB) e a área de gordura do braço (AGB), respectivamente, utilizando-se os parâmetros de Frisancho. ${ }^{(15)} \mathrm{A}$ análise foi realizada utilizando-se a adequação percentual em relação ao percentil 50, definindo-se como com déficit nutricional os pacientes que apresentassem adequação < 90\% e, como eutróficos, aqueles com adequação $\geq 90 \%$.

Para a avaliação dos indicadores nutricionais, foi utilizado o programa WHO Anthro 2005 (OMS, Genebra, Suíça). ${ }^{(16)}$ Utilizou-se o teste de

Tabela 1 - Dados antropométricos e escore de Shwachman, por faixa etária, expresso em média e dp, em fibrocísticos atendidos em um centro de referência em Recife (PE).

\begin{tabular}{lcccc}
\hline \multicolumn{1}{c}{ ldade (anos) } & $0 \dashv 5$ & $5 \dashv 10$ & $10 \dashv 18$ & $\mathrm{p}^{*}$ \\
\hline $\mathrm{n}$ & 7 & 6 & 8 & $\mathrm{NS}$ \\
$\mathrm{P} / \mathrm{l}$ & $-0,73 \pm 1,26$ & $-0,75 \pm 1,00$ & $-0,89 \pm 1,13$ & $\mathrm{NS}$ \\
$\mathrm{A} / \mathrm{l}$ & $-0,18 \pm 1,71$ & $-0,39 \pm 0,93$ & $-0,52 \pm 1,56$ & $\mathrm{NS}$ \\
$\mathrm{P} / \mathrm{A}^{\mathrm{a}}$ & $-0,75 \pm 0,96$ & $-0,74 \pm 0,90$ & - & $\mathrm{NS}$ \\
$\% \mathrm{P} / \mathrm{A}$ & $95,14 \pm 5,83$ & $95,97 \pm 8,37$ & $92,68 \pm 21,41$ & 0,013 \\
$\mathrm{AMB} / 0^{\mathrm{b}}$ & - & $35,41 \pm 12,33$ & $64,30 \pm 16,34$ & $\mathrm{NS}$ \\
$\mathrm{AGB} \% \mathrm{o}^{\mathrm{b}}$ & - & $49,66 \pm 17,76$ & $59,97 \pm 36,48$ & $\mathrm{NS}$ \\
Escore de Shwachman & $81,67 \pm 87,51$ & $82,50 \pm 16,05$ & $81,43 \pm 11,44$ &
\end{tabular}

P/l: peso/idade; A/l: altura/idade; P/A: peso/altura; \%P/A: \%peso ideal/altura; AMB: área muscular do braço; AGB: área gordurosa do braço; NS: não significativo. ${ }^{a}$ Medidas realizadas em menores de 10 anos; ${ }^{b}$ Medidas realizadas em maiores de 5 anos. "Teste one-way ANOVA. 
normalidade de Kolmogorov-Smirnov, e a distribuição apresentou-se simétrica, sendo os dados apresentados como média e dp. 0 teste $t$ de Student foi utilizado para comparar dois grupos independentes, e foi aplicado o teste one-way ANOVA para mais de dois grupos. Para a comparação de proporções foi utilizado o teste exato de Fisher. Adotou-se um nível de significância de 0,05 .

O estudo foi aprovado pelo Comitê de Ética em Pesquisa do IMIP e, posteriormente, foi esclarecido com detalhes aos pais ou responsáveis que, após concordarem, assinaram um termo de consentimento autorizando a participação do paciente no estudo.

\section{Resultados}

Foram coletados dados referentes a 21 indivíduos, dos quais $12(57,1 \%)$ eram do sexo feminino e $9(42,9 \%)$ do sexo masculino, com idade média de 8,4 \pm 4,6 anos, sendo a distribuição por faixa etária dividida em três grupos: 7 (33,3\%) menores de 5 anos, $6(28,6 \%)$ com idade entre 5 e 10 anos e $8(38,1 \%)$ maiores de 10 anos.

Quanto à procedência dos indivíduos, a maioria $(52,3 \%)$ residia na região metropolitana do Recife, seguida do interior do estado de Pernambuco $(42,9 \%)$, e 1 paciente $(4,8 \%)$ era proveniente de outro estado, Paraíba.

A idade de diagnóstico variou desde o período neonatal até 14 anos, com média de 3,8 $\pm 3,9$ anos, e o diagnóstico foi realizado após os 10 anos de idade em 9,5\% dos casos. As principais manifestações clínicas presentes ao diagnóstico foram as seguintes: sintomas respiratórios agudos ou persistentes (85,7\%), esteatorreia $(66,7 \%)$ e déficit nutricional $(47,6 \%$; Figura 1). Quatro pacientes $(19,1 \%)$ tinham história positiva de FC na família.

As médias dos indicadores nutricionais $(\mathrm{P} / 1$, $\mathrm{A} / 1$ e $\mathrm{P} / \mathrm{A})$ das faixas etárias encontraram-se dentro dos limites para eutrofia, considerado maior que -1 escore $\mathrm{Z}$ para os parâmetros avaliados. De uma forma geral, os menores de 5 anos apresentaram melhores indicadores do estado nutricional quando comparados aos outros grupos. Houve um maior comprometimento do estado nutricional nos indivíduos maiores de 10 anos em relação ao \% $\mathrm{P} / \mathrm{A}$, mas não houve diferença estatisticamente significante entre as faixas etárias para ambos os indicadores, com $p>0,05$, conforme Tabela 1 .
Os resultados divergiram dos apresentados pelos indicadores antropométricos quanto à composição corporal. Houve déficit acentuado tanto em relação à massa corporal magra, representada pela $\mathrm{AMB}$, quanto do compartimento de gordura, representado pela AGB, com médias abaixo de $60 \%$ de adequação para a eutrofia, demonstrando depleção severa das reservas corpóreas e sendo mais pronunciada na faixa etária entre 5 e 10 anos, com diferença estatisticamente significante para a AMB (Tabela 1).

0 percentual de nutridos e desnutridos variou conforme o parâmetro utilizado para classificação do estado nutricional. Utilizando-se os parâmetros da OMS (escore Z), ${ }^{(11,12)}$ foram diagnosticados como desnutridos 66,7\% dos indivíduos, enquanto que, quando os mesmos foram avaliados pela recomendação dos consensos de nutrição em FC $(\% \mathrm{P} / \mathrm{A}),{ }^{(13,14)}$ apenas 33,3\% apresentavam-se distróficos. Com o interesse em verificar a existência ou não de diferenças significantes entre a classificação de desnutrição (déficit nutricional) e eutrofia, foi aplicado o teste exato de Fisher, resultando em diferença não-significativa, com $p>0,05$ (Tabela 2).

Quando avaliado o estado nutricional de acordo com a idade de diagnóstico, os indivíduos identificados com menos de um ano de vida apresentaram melhores indicadores nutricionais em relação aos diagnosticados com maior faixa etária, sem diferenças estatísticas significantes.

Segundo o escore de Shwachman, 52,6\% e $47,4 \%$ dos pacientes apresentaram gravidade leve e moderada para a doença, respectivamente. Não houve registro de severidade em relação a esse escore. Três pacientes (14,3\%), menores de 10 anos, apresentaram classificação excelente (100 pontos), indicando o melhor estado clínico em crianças mais jovens, apesar de não

Tabela 2 - Classificação do estado nutricional, segundo a Organização Mundial de Saúde e consensos de nutrição em FC, em fibrocísticos atendidos em um centro de referência no Recife (PE).

\begin{tabular}{lll}
\hline \multicolumn{1}{c}{ Estado nutricional } & $\begin{array}{r}\text { OMS, } \\
\mathrm{n}(\%)\end{array}$ & $\begin{array}{c}\text { Consensos, } \\
\mathrm{n}(\%)\end{array}$ \\
\hline Sem déficit nutricional & $7(33,3)$ & $14(66,7)$ \\
Com déficit nutricional & $14(66,7)$ & $7(33,3)$ \\
Total & $21(100)$ & $21(100)$ \\
\hline
\end{tabular}

OMS: Organização Mundial de Saúde; Teste Exato de Fisher $(p>0,05)$. 
Tabela 3 - Características neonatais, socioeconômicas e clínicas, segundo o estado nutricional de fibrocísticos atendidos em um centro de referência no Recife (PE).

\begin{tabular}{|c|c|c|c|}
\hline & $\begin{array}{l}\text { Sem déficit } \\
\text { nutricional }^{\text {a }}\end{array}$ & $\begin{array}{l}\text { Com déficit } \\
\text { nutricional }^{\mathrm{b}}\end{array}$ & $p$ \\
\hline $\mathrm{n}$ & 7 & 14 & \\
\hline Idade (anos) & $6,94 \pm 5,11$ & $8,64 \pm 4,45$ & $\mathrm{NS}^{*}$ \\
\hline \multicolumn{4}{|l|}{ Características neonatais } \\
\hline Peso ao nascer (g) & $3.139 \pm 512$ & $3.351 \pm 903$ & $\mathrm{NS}^{*}$ \\
\hline Tempo de aleitamento materno exclusivo (meses) & $3,33 \pm 1,16$ & $2,62 \pm 2,47$ & NS \\
\hline \multicolumn{4}{|l|}{ Características socioeconômicas } \\
\hline Idade materna (anos) & $36,50 \pm 7,94$ & $31,92 \pm 7,35$ & NS* \\
\hline Anos de estudo materno (anos) & $11,33 \pm 2,42$ & $9,00 \pm 4,73$ & $\mathrm{NS}^{*}$ \\
\hline Número de filhos & $2,86 \pm 1,22$ & $2,31 \pm 0,86$ & $\mathrm{NS}^{*}$ \\
\hline Número de residentes no domicílio & $5,00 \pm 2,31$ & $4,31 \pm 1,11$ & NS \\
\hline Renda per capita (R\$) & $407,88 \pm 395,15$ & $226,46 \pm 199,48$ & $\mathrm{NS}^{*}$ \\
\hline \multicolumn{4}{|l|}{ Características clínicas } \\
\hline ldade de diagnóstico (anos) & $2,44 \pm 3,48$ & $4,52 \pm 3,98$ & $\mathrm{NS}^{*}$ \\
\hline Escore de Shwachman & $94,29 \pm 5,35$ & $74,58 \pm 12,52$ & $0,001^{*}$ \\
\hline Colonização por $P$. aeruginosa & $28,6 \%$ & $71,4 \%$ & $\mathrm{NS}^{* \prime \prime}$ \\
\hline Colonização por $S$. aureus oxacilina sensivel & $33,3 \%$ & $66,7 \%$ & $\mathrm{NS}^{* *}$ \\
\hline Colonização por S. aureus oxacilina resistente & $33,3 \%$ & $66,7 \%$ & $\mathrm{NS}^{\prime \prime}$ \\
\hline Colonização por B. cepacia & $25,0 \%$ & $75,0 \%$ & $\mathrm{NS}^{\prime \prime \prime}$ \\
\hline
\end{tabular}

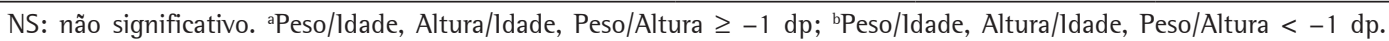
*Teste t de Student; **teste exato de Fisher.

ter havido diferença significante entre as faixas etárias (Tabela 1).

As características neonatais, socioeconômicas e clínicas foram avaliadas segundo o estado nutricional (nutridos $v S$. com déficit nutricional), com base no escore $\mathrm{Z}$, e encontram-se na Tabela 3. Apesar de não ter havido diferença estatisticamente significante entre os grupos, os indivíduos que tiveram maior tempo de aleitamento materno exclusivo, bem como maior idade materna, maior escolaridade materna e maior renda per capita, apresentaram estado nutricional melhor. Quanto às características clínicas, os indivíduos eutróficos foram diagnosticados mais precocemente e apresentaram menor número de colonizações pulmonares dentre os microorganismos avaliados. A média do escore de Shwachman foi significativamente maior nos pacientes eutróficos, demonstrando a influência do estado nutricional na determinação desse índice.

\section{Discussão}

A literatura é escassa em publicar dados socioeconômicos de indivíduos portadores de FC, não sendo possível comparar nossos resultados com as realidades sociais de outras localidades brasileiras. Diante disso, destaca-se a importância do estudo dessas variáveis para o conhecimento das peculiaridades sociais dessa população.

0 melhor estado nutricional encontrado em nossa casuística pode ser explicado pelas condições neonatais e socioeconômicas favoráveis.

0 peso ao nascer e o aleitamento materno são importantes preditores do estado nutricional. A idade gestacional e o adequado peso ao nascer, juntamente com o tempo de aleitamento materno, atuam como fatores protetores contra a ocorrência de doenças frequentes, principalmente as de origem infecciosa e a desnutrição, ${ }^{(17)}$ o que pode ter contribuído para o melhor estado nutricional apresentado pelos indivíduos estudados, quando comparado a outros estudos. ${ }^{(18-20)}$

Com relação à escolaridade materna, o maior número de anos de estudo favorece o melhor estado nutricional, bem como melhora a adesão ao tratamento dos pacientes. A renda familiar pode ser considerada um importante determinante das condições de saúde pela influência que exerce na possibilidade de aquisição e utilização de bens e serviços essenciais à manutenção 
do estado de saúde, tais como alimentação, moradia, vestuário e saneamento. ${ }^{(21)}$

A média da idade de diagnóstico em nossa casuística foi semelhante à de outro estudo realizado no sudeste brasileiro. ${ }^{(22)}$ No entanto, foi relatado que a mediana da idade de diagnóstico nos EUA foi de 6 meses. ${ }^{(23)}$ Em nosso estudo, os pacientes eutróficos apresentaram menor média de idade de diagnóstico quando comparado aos com déficit nutricional, demonstrando a necessidade do diagnóstico precoce, principalmente com a implementação da triagem neonatal, que ainda é pouco realizada em nossa região. 0 diagnóstico realizado precocemente é importante para a profilaxia das infecções pulmonares e reposição de enzimas pancreáticas nos insuficientes pancreáticos, melhorando a absorção de nutrientes com o objetivo de manter o bom estado nutricional, diminuindo as complicações resultantes da doença e aumentando a sobrevida. ${ }^{(24)}$

As manifestações clínicas podem ser muito variáveis e ocorrerem precocemente ou se manifestarem mais tardiamente. As mais comuns ao diagnóstico na FC descritas na literatura são a tosse persistente, diarreia crônica e déficit nutricional, ${ }^{(22,25,26)}$ como observado em nosso estudo. 0 escore de Shwachman demonstrou boas condições clínicas em nossa casuística, com média de $81,8 \pm 14,2$, enquanto outros estudos apresentaram médias de $74 \pm 17^{(26)}$ e 56 $\pm 15,{ }^{(27)}$ este em pacientes internados, evidenciando maior agravamento clínico.

Em estudos nacionais ${ }^{(19,20)}$ e internacionais, ${ }^{(5,18)}$ relatou-se algum grau de desnutrição em pacientes portadores de FC, bem como maior comprometimento do estado nutricional em indivíduos com idade superior a 10 anos. Nesses estudos atribuiu-se tal fato ao aumento das necessidades nutricionais devido ao crescimento acelerado ou ao aumento do gasto energético relacionado às infecções pulmonares, que são mais frequentes, e a alterações na função endócrina, pela maior probabilidade de aparecimento de diabetes nessa faixa etária. ${ }^{(28)}$ Também pode haver uma menor adesão à terapia de reposição enzimática, mudanças na dieta e aumento da atividade física nesta faixa etária. ${ }^{(20)}$ Vale salientar que todos os indivíduos estudados apresentavam insuficiência pancreática e faziam uso adequado e regular de enzimas pancreáticas. Nenhum deles tinha o diagnóstico de diabetes melito.
0 déficit de massa corporal em fibrocísticos geralmente acontece nos compartimentos de gordura corporal e massa magra, o que pode estar associado a um aumento significativo no catabolismo das proteínas e da reserva adiposa que ocorre na FC. 0 balanço energético e o metabolismo proteico são afetados adversamente pelos episódios de exacerbação aguda de infecções pulmonares, o que prejudica a função dos músculos respiratórios, exercícios de tolerância e função imune, com piora do prognóstico destes pacientes. ${ }^{(20,29)}$

Ao avaliarmos o estado nutricional, houve divergência quando utilizados os padrões propostos pela OMS e os recomendados pelos consensos internacionais de FC. Além disso, a avaliação da composição corporal apresentou uma redução no número de indivíduos considerados eutróficos e um aumento dos casos de desnutrição. Tal fato também foi identificado em outro estudo. ${ }^{(30)}$

Podemos concluir que os pacientes atendidos neste centro apresentaram estado nutricional variado conforme os parâmetros utilizados para a classificação, tendendo à eutrofia em relação aos indicadores antropométricos (peso e altura) e ao déficit nutricional quando avaliados os compartimentos muscular e de gordura, demonstrando a necessidade de uma avaliação nutricional específica e mais detalhada que englobe todos esses indicadores. As condições neonatais e socioeconômicas apresentaram-se favoráveis, principalmente em relação ao grau de instrução materna e renda per capita, que contribuem de forma direta para uma melhor adesão ao tratamento e relacionando-se com o melhor estado nutricional. Em relação às condições clínicas, a menor idade de diagnóstico, menor número de infecções pulmonares e melhor escore de Shwachman foram encontrados nos individuos com estado nutricional mais preservado.

\section{Referências}

1. Reis FJ, Damaceno N. Fibrose Cística. J Pediatr (Rio J). 1998;74(Supl 1):S76-S94.

2. Ribeiro JD, Ribeiro MA, Ribeiro AF. Controvérsias na fibrose cística - do pediatra ao especialista. J Pediatr (Rio J). 2002;78(Supl 2):S171-86.

3. Raskin S, Phillips JA 3rd, Krishnamani MR, VnencakJones C, Parker RA, Rozov T, et al. DNA analysis of cystic fibrosis in Brazil by direct PCR amplification from Guthrie cards. Am J Med Genet. 1993;46(6):665-9.

4. Santos GP, Domingos MT, Wittig EO, Riedi CA, Rosário NA. Programa de triagem neonatal para fibrose cística 
no estado do Paraná: avaliação após 30 meses de sua implantação. J Pediatr (Rio J). 2005;81(3):240-4.

5. Lai HC, Kosorok MR, Sondel SA, Chen ST, FitzSimmons SC, Green CG, et al. Growth status in children with cystic fibrosis based on the National Cystic Fibrosis Patient Registry data: evaluation of various criteria used to identify malnutrition. J Pediatr. 1998;132(3 Pt 1):478-85.

6. Bentur L, Kalnins D, Levison H, Corey M, Durie PR. Dietary intakes of young children with cystic fibrosis: is there a difference? J Pediatr Gastroenterol Nutr. 1996;22(3):254-8.

7. Creveling S, Light M, Gardner P, Greene L. Cystic fibrosis, nutrition, and the health care team. J Am Diet Assoc. 1997;97(10 Suppl 2):S186-S91.

8. Gaspar MC, Chiba SM, Gomes CE, Juliano Y, Novo NF, Ancona-Lopez F. Resultado de intervenção nutricional em crianças e adolescentes com fibrose cística. J Pediatr (Rio J). 2002;78(2):161-70.

9. Smyth RL. Diagnosis and management of cystic fibrosis. Arch Dis Child Educ Pract Ed. 2005;90:1-6.

10. Santos $\mathrm{Cl}$, Ribeiro JD, Ribeiro AF, Hessel G. Análise crítica dos escores de avaliação de gravidade da fibrose cística: estado da arte. J Bras Pneumol. 2004;30(3):286-98.

11. World Health Organization [homepage on the Internet]. Geneva: World Health Organization. [cited 2007 Jan 20]. The WHO Child Growth Standards. Avaliable from: http://www.who.int/childgrowth/standards/en/

12. Hamill PV, Drizd TA, Johnson CL, Reed RB, Roche AF; Department of Health Education And Welfare Washington DC. NCHS Growth Curves for Children Birth-18 Years. Myattsville: NCHS; 1977.

13. Ramsey BW, Farrell PM, Pencharz P. Nutritional assessment and management in cystic fibrosis: a consensus report. The Consensus Committee. Am J Clin Nutr. 1992;55(1):108-16.

14. Borowitz D, Baker RD, Stallings V. Consensus report on nutrition for pediatric patients with cystic fibrosis. J Pediatr Gastroenterol Nutr. 2002;35(3):246-59.

15. Frisancho AR. New norms of upper limb fat and muscle areas for assessment of nutritional status. Am J Clin Nutr. 1981;34(11):2540-5.

16. World Health Organization [homepage on the Internet]. Geneva: World Health Organization. [cited 2007 May 25]. WHO Anthro 2005 software and macros; [about 2 screens]. Available from: http://www.who.int/ childgrowth/software/en/index.html

17. Spyrides MH, Struchiner CJ, Barbosa MT, Kac G. Efeitos das práticas alimentares sobre o crescimento infantil. Rev Bras Saúde Matern Infant. 2005;5(2):145-53.
18. Morison S, Dodge JA, Cole TJ, Lewis PA, Coles EC, Geddes $D$, et al. Height and weight in cystic fibrosis: a cross sectional study. UK Cystic Fibrosis Survey Management Committee. Arch Dis Child. 1997;77(6):497-500.

19. Fiates GM, Barbosa E, Auler F, Feiten SF, Miranda F. Estado nutricional e ingestão alimentar de pessoas com fibrose cística. Rev Nutr (Campinas). 2001;14(2):95-101.

20. Adde FV, Rodrigues JC, Cardoso AL. Seguimento nutricional de pacientes com fibrose cística: papel do aconselhamento nutricional. J Pediatr (Rio J). 2004;80(6):475-82.

21. Castro TG, Novaes JF, Silva MR, Costa NM, Franceschini SC, Tinôco AL. Caracterização do consumo alimentar, ambiente socioeconômico e estado nutricional de pré-escolares de creches municipais. Rev Nutr (Campinas). 2005;18(3):321-30.

22. Alvarez AE, Ribeiro AF, Hessel G, Bertuzzo CS, Ribeiro JD. Fibrose cística em um centro de referência no Brasil: características clínicas e laboratoriais de 104 pacientes e sua associação com o genótipo e a gravidade da doença. J Pediatr (Rio J). 2004;80(5):371-9.

23. Cystic Fibrosis Foundation. Annual Data Report 2002. Bethesda (MD): CFF; 2003.

24. Campos JV, Damaceno N, Carvalho CR, Kotze LM. Fibrose Cística. Arq Gastroenterol. 1996;33:1-48.

25. Farias L, Rosário Filho NA, Kovalhuk L, Miasaki N, Chaves SM, Recco RA, et al. Aspectos clínicos da fibrose cística: experiência no Hospital de Clínicas da UFPR, 1980-1996. Pediatria (São Paulo). 1997;19(4):241-8.

26. Reis FJ, Oliveira MC, Penna FJ, Oliveira MG, Oliveira EA, Monteiro AP. Quadro clínico e nutricional de pacientes com fibrose cística: 20 anos de seguimento no HC-UFMG. Rev Assoc Med Brasil. 2000;46(4):325-30.

27. Adde FV, Dolce P, Tanikawa CE, Uehara DY, Cardoso AL, Rozov T. Suplementação dietética em pacientes com fibrose cística. J. Pediatr (Rio J). 1997;73(5):317-23.

28. Alves CA, Aguiar RA, Alves ACS, Santana MA. Diabetes mellitus in patients with cystic fibrosis. J Bras Pneumol. 2007;33(2):213-21.

29. Hart N, Tounian P, Clément A, Boulé M, Polkey Ml, Lofaso F, et al. Nutritional status is an important predictor of diaphragm strength in young patients with cystic fibrosis. Am J Clin Nutr. 2004;80(5):1201-6.

30. Morlin C, Gomes LS, Morsoletto RH, Pereira LC. Perfil nutricional dos pacientes atendidos no ambulatório de fibrose cística de um hospital público de Uberlândia Minas Gerais. Rev Bras Nutr Clin. 2006;21(1):12-6.

\section{Sobre os autores}

Isabel Carolina da Silva Pinto

Nutricionista. Instituto Materno Infantil Professor Fernando Figueira - IMIP - Recife (PE) Brasil.

Cristiane Pereira da Silva

Nutricionista. Instituto Materno Infantil Professor Fernando Figueira - IMIP - Recife (PE) Brasil. 